\title{
Computer-aided Measurement of Partial Discharges in HV Equipment
}

\author{
E. Gulski \\ HV Laboratory, Electrical Engineering Department, \\ Delft University of Technology, The Netherlands
}

\begin{abstract}
In this paper the previously developed method of partial discharges (PD) recognition is applied to evaluate $P D$ in $H V$ devices. Using conventional discharge detection (bandwidth $\sim 400 \mathrm{kHz}$ ) the patterns of PD were studied. Satisfactory results in the past, when analyzing discharges in artificial models, have shown that using the shape of phase-position distributions the origin and the type of discharges can be studied. By means of unambiguous statistical operators like skewness, kurtosis, number of peaks etc. quantitative information about the discharge source can be obtained. In the case of an unknown defect a combination of these operators is compared to the same set of operators for a known configuration. In this way a statement can be made on the character of a discharge source. The use of this technique in evaluating different discharges in $\mathrm{HV}$ cable and in $400 \mathrm{kV} \mathrm{SF} 6$ insulation confirms the usefulness of the new approach.
\end{abstract}

\section{INTRODUCTION}

$\mathrm{D}$ SCHARGE detection is an important tool for evaluating $\mathrm{HV}$ insulating system constructions. In practice the evaluation is restricted to comparing inception voltage (in $\mathrm{kV}$ ) and largest discharge magnitude (in $\mathrm{pC}$ ) with the test specifications. Often, if the maximum allowable discharge level is exceeded, it is important to know the cause of the discharge. Therefore, commercial PD measuring systems provide oscillographic observation in accordance with a method published by CIGRE in 1969 [1]. However, the interpretation of a PD pattern from an oscilloscope often leaves room for considerable doubt and provides only limited recognition of the defects causing discharges. An example of patterns that undergo change over a short time is shown in Figure 1. These three oscillographic PD patterns were made during a 20 min test, observing discharges in a spacer of a $400 \mathrm{kV} \mathrm{SF}_{6}$ compartment of a gas-insulated substation system (GIS). Furthermore, the oscillographic method requires an experienced observer and can be used during the test only. Therefore, investigation into the use of digital techniques for the evaluation of PD has become increasingly important; this trend concentrates on computer-assisted judgment of the quality and the condition of insulating systems [2]. The main goal here is to answer the questions: "How to correlate the measured discharges to different discharge sources?" and "Do the discharges decrease the life expectancy of the device?" While it is very difficult to answer the second question, even before it can be answered, a solution to the problem in the first question must be found. Although it may be possible to recognize different defects, in general we are still not able to say whether defect $A$ is harmful and defect $B$ is not. The moment that we are able to make this statement for a wide range 


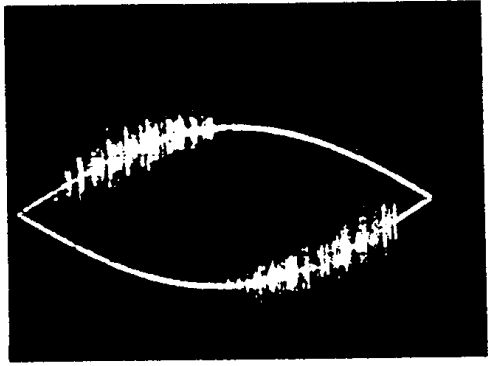

Oscillographic patterns of PD made on a spacer in a $400 \mathrm{kV}$ GIS compartment. Object was subjected to a $250 \mathrm{kV}$ test voltage during $20 \mathrm{~min}$.

of dielectrics and configurations, the time has come to review the standard test specifications.

According to recent developments in this area, much effort has been concentrated on automation of tests for cables, power transformers, rotating electrical machines, generators and other insulated devices. It is known that a recording of a complete a measurement permits a more systematic analysis of a process as well as its control. Especially when in the case of PD this process is very complex and characterized by stochastic behavior $[3,4]$.

In recent years, with the use of digital processing, a renewed interest has developed for the observation of discharges as a function of the phase angle. Twentyfour years ago it was shown that phase-resolved observation of PD represents a means for evaluating different discharge sources [5]. Each discharge source is characterized by a specific sequence of discharges due to its geometry, location in insulation, dielectric properties and applied electric field. Especially with the introduction of computeraided processing, this evaluation can be improved when phase-position quantities are used.

\section{ANALYSIS OF PD}

\subsection{ACQUISITION OF PD}

An analyzer has been constructed whereby the evaluation of phase-position quantities is automated [6]. This system consists of a PD detection circuit, a data acquisition unit and a personal computer as shown in Figure 2. To measure PD, a commercial PD detector, Haefely Type 560 , is used. Its bandwidth has a lower limit of $40 \mathrm{kHz}$ and an upper limit of $400 \mathrm{kHz}$. According to IEC 270 the $\mathrm{PD}$ pulses are integrated such that the maximum value

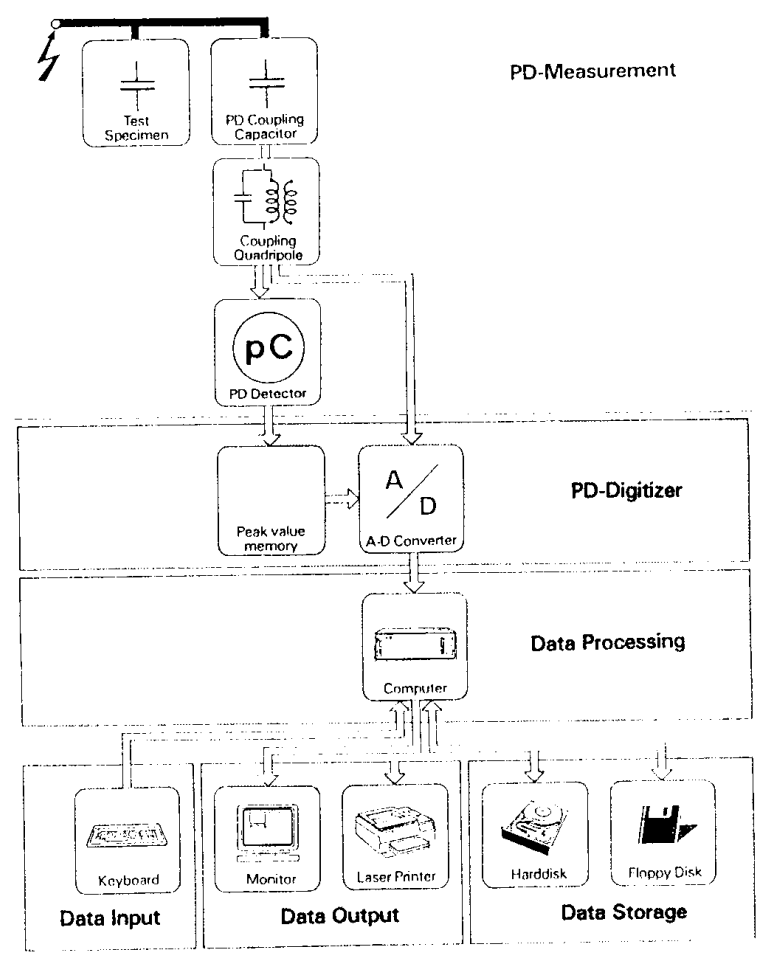

Figure 2.

Block diagram of the discharge analyzer.

of the integrated signal is proportional to the apparent charge. Therefore, a peak value memory (sample and hold) was developed to register continuously all PD peak values [7]. The acquisition time of the peak value storage was adjusted to the PD detector bandwidth. The acquisition time of the peak value storage was adjusted to the lower and upper cutoff frequencies of the detector: $5 \mu \mathrm{s}$ at a bandwidth of $500 \mathrm{kHz}$ and $\approx 150 \mu \mathrm{s}$ at a bandwidth of $10 \mathrm{kHz}$.

In order to prevent recording of system noise, a noise suppression of $10 \%$ was employed throughout the PD 


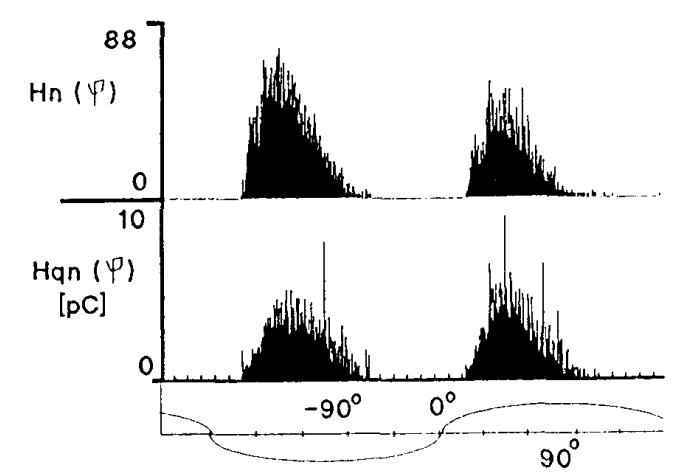

(a)

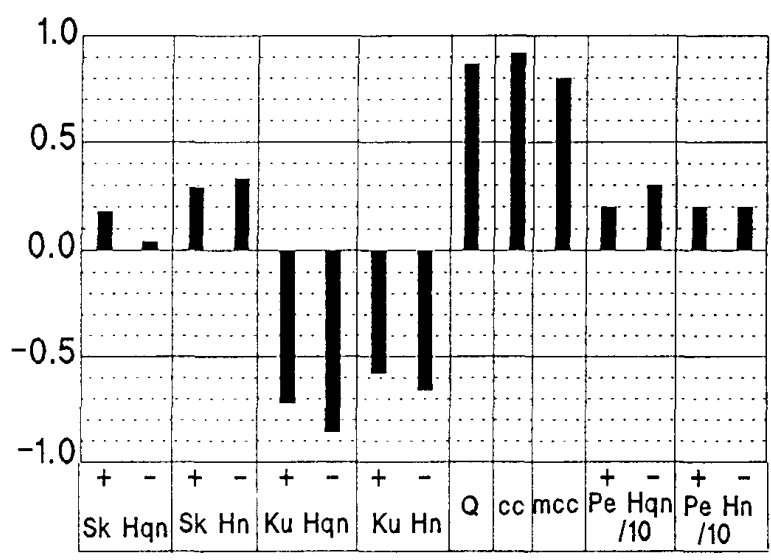

(b)

Figure 3.

Example of analysis for spacer discharges in a $400 \mathrm{kV}$ GIS compartment obtained at $250 \mathrm{kV}$ test voltage during $10 \mathrm{~min}$ test: the pulse count distribution $H_{n}(\varphi)$ and the mean pulse height distribution $H_{q n}(\varphi)$; (b) statistical operators processed for the $H_{n}(\varphi)$ and $H_{q n}(\varphi)$ as shown in (a).

(a) Skewness Sk of Hqn ( $\varphi)$

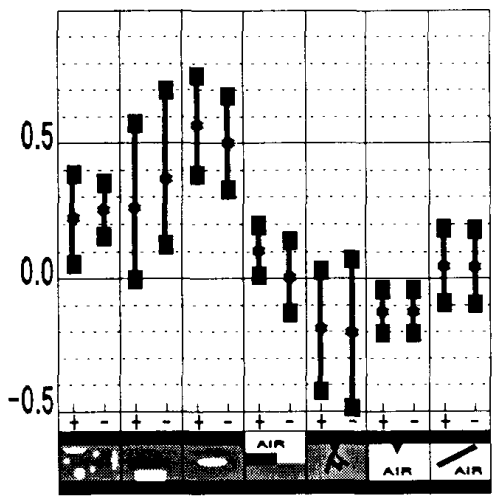

(b) Kurtosis $\mathrm{Ku}$ of $\mathrm{Hn}(\varphi)$

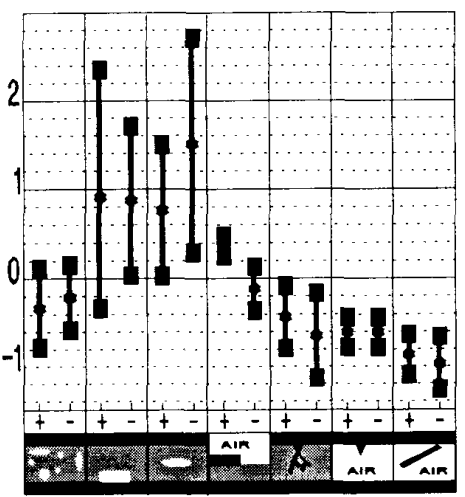

Figure 4. (c) mec factor of Hqn ( $\varphi$

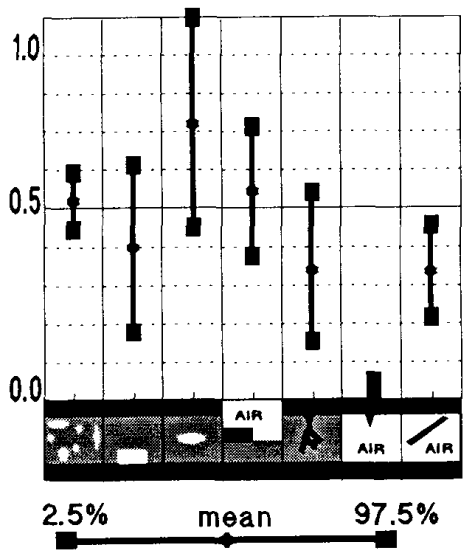

Comparison of values of one and the same statistical operator as obtained for the 7 different defects:

(a) skewness $S k$ of $H_{q^{n}}^{ \pm}(\varphi)$, (b) kurtosis $K u$ of $H_{n}^{ \pm}(\varphi)$ and (c) mcc factor of $H_{q n}(\varphi)$.

tests. Therefore, only signals $>10 \%$ of the maximum discharge amplitude were analyzed. To synchronize the PD signal with the $50(60) \mathrm{Hz}$ time base and to trigger the acquisition unit, a HV sine wave was tapped off from the coupling capacitor and digitized by a $12 \mathrm{kHz}$ sampling frequency.

To identify the measured $\mathrm{PD}$ in $\mathrm{pC}$, the system was calibrated. To that effect, a known discharge was injected across the specimen, and a discharge resolution factor was calculated representing the smallest difference in the discharge magnitude $(>1 \mathrm{pC})$ that can be detect- ed. To process the data, an industrial personal computer containing an Intel $80386 \mathrm{CPU}$ with a $33 \mathrm{MHz}$ clock frequency and 120 Mbyte hard disk was used. To provide during the measurement a continuous transfer of PD to the computer, the software routines of processing, storage and displaying were written in Turbo Assembler 2.0. The routines to process the data after completion of the measurements were written in Turbo Pascal 6.0. 


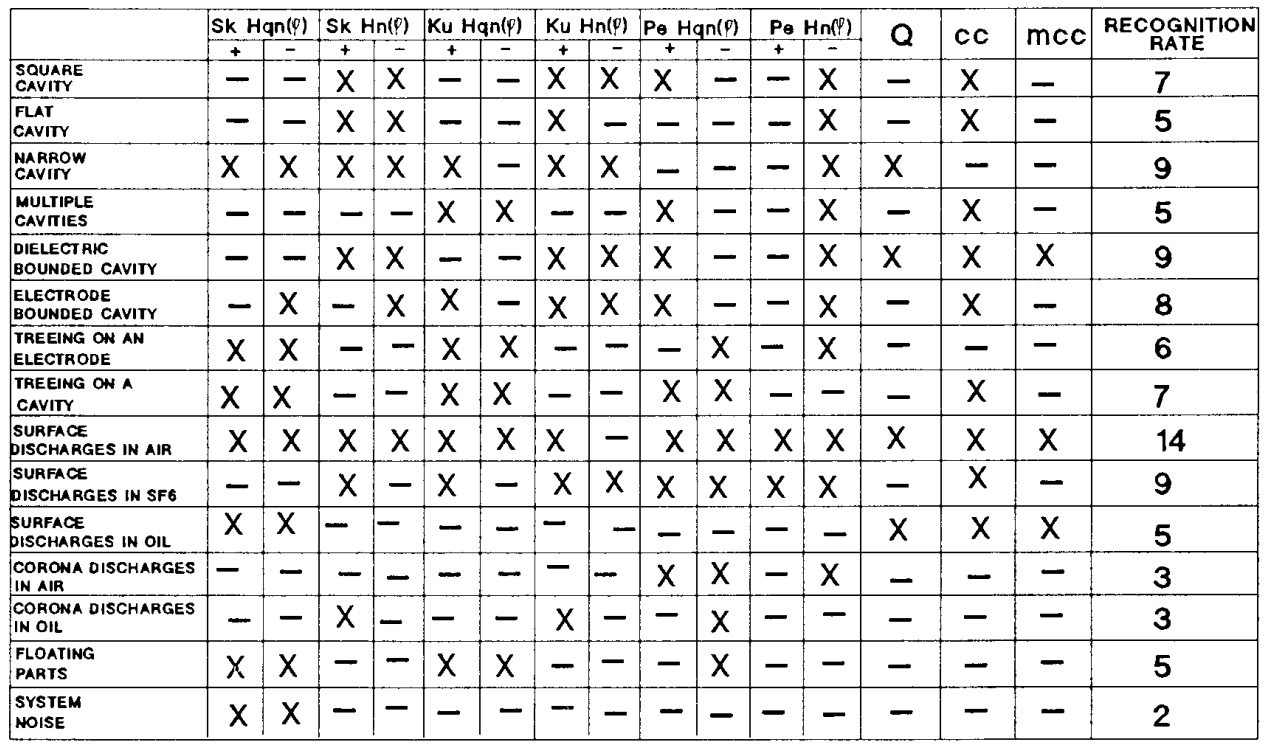

X : statistical operator fits in the finger print of a defect

: statistical operator does not fit in the finger print of a defect

(a)

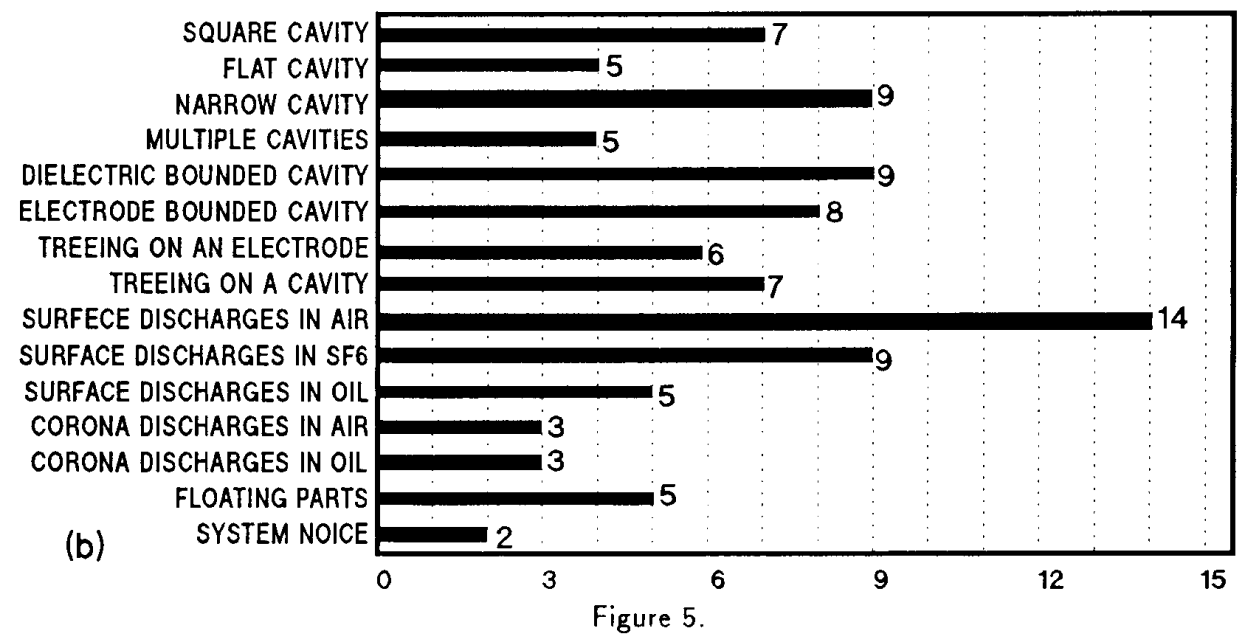

Example of recognition rate of an unknown defect with the aid of the statistical operators: (a) matrix for comparison of an unknown discharge source with the finger print of 15 known discharge sources, (b) graphic output of the recognition rate processed in (a).

\subsection{PHASE-RESOLVED PD DISTRIBUTIONS}

Because of the phase-dependent behavior of PD generated under ac voltages conditions, several quantities as function of the phase angle can be used to describe the PD phenomena. For this purpose the voltage cycle should be divided into phase windows representing the phase angle axis $\left(0\right.$ to $\left.360^{\circ}\right)$. If the observation takes place over several voltage cycles, in each phase window the statistical distribution of individual PD events can be determined [4]. Finally, these distributions observed throughout the whole angle axis result in a three-dimensional distribution. Such three-dimensional distributions are attractive for the eye; however because of theirs complexity, such presentations are more difficult to analyze. In addition, due to the dependence of the PD phenomena on the field strength and the time during which the voltage is applied, these distributions become increasingly more 


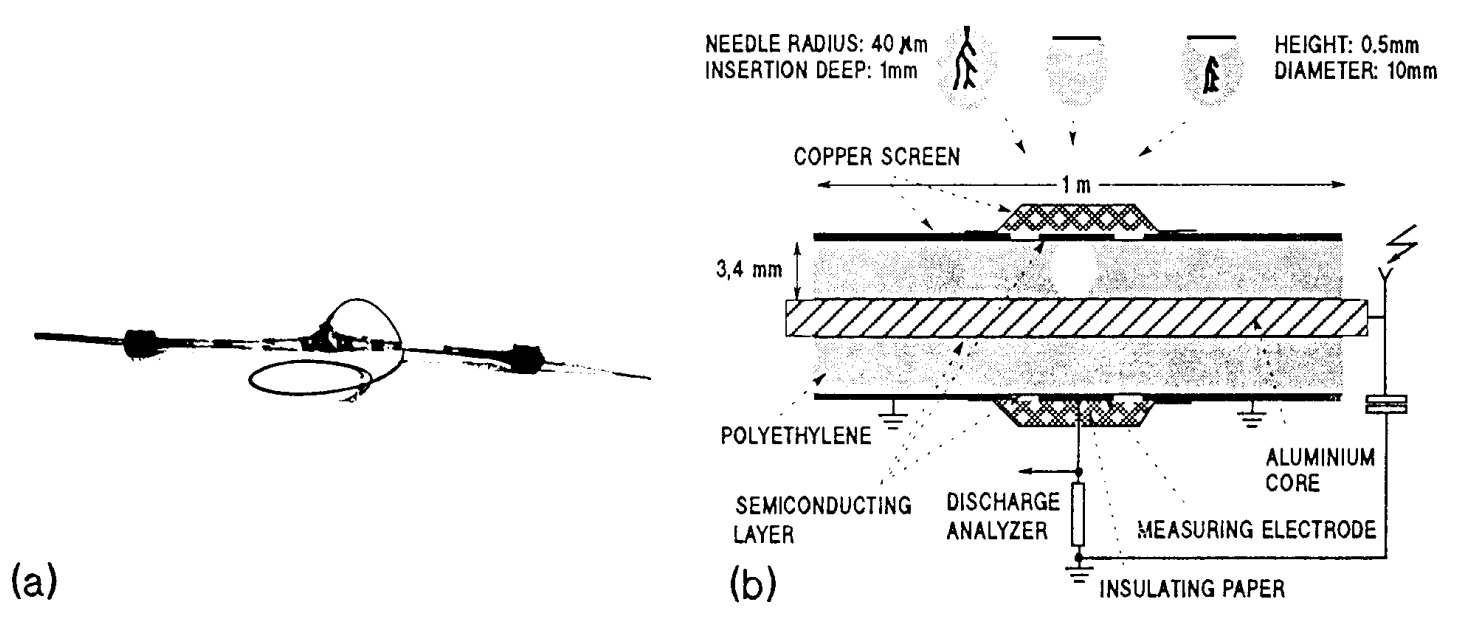

Figure 6.

Test sample. (a) photograph of the $6 / 10 \mathrm{kV}$ PE cable, (b) cross section of the cable showing the artificial defects.

complex. For convenience of comparison, the choice has been made by the author to use two-dimensional distributions. Moreover, the latter distributions can be derived from statistical distributions as mentioned above by taking appropriate averages. For this purpose in each phase window three quantities are determined:

1. the sum of the discharge magnitudes observed in one phase window, $q_{\mathrm{s}}=\sum q_{i}$

2. the number of discharges observed in one phase window, $n=\sum i$

3. the average value of discharges observed in one phase window, $q_{n}=q_{s} / n$

where $q_{i}$ is the discharge magnitude in a phase window during one voltage cycle, and $i=1$ if a discharge pulse occurs in the phase window, $i=0$ if no discharge pulse occurs. These quantities observed throughout the whole angle axis result in the following two distributions as function of the phase angle $\varphi_{i}$.

1. The pulse count distribution $H_{n}(\varphi)$ which represents the number of the observed discharges in each phase window as a function of the phase angle.

2. The mean pulse height distribution $H_{q n}(\varphi)$ which represents the average amplitude in each phase window as a function of the phase angle. $H_{q n}(\varphi)$ is derived from the total discharge amount in each phase window divided by the number of discharges in the same phase window.

Consequently, computer-aided processing starts by the grouping of discharge impulses according to their phaseposition of the $50(60) \mathrm{Hz}$ sinewave. A number of phase windows 4000 for $50 \mathrm{~Hz}$ and 3333 for $60 \mathrm{~Hz}$ sinewave is established and each phase window is observed for a large number of cycles, for instance during $\sim 5 \mathrm{~min}$. Its known, that due to the nonstationary nature of the PD phenomena, the distribution profiles can vary for one and the same type of discharge source. To provide reproducibility of the measured distributions, the duration of the test and the level of test voltages are important. Therefore, the phase-position distributions of artificial defects as discussed in $[6,8]$ are measured during a 20 min test at $20 \%$ above the discharge inception voltage. Experience has shown that a $20 \mathrm{~min}$ test period is sufficient to reveal, in the case of PD in solid materials, the properties of a discharge.

An example of these two distributions is shown in Figure 3(a). As shown in this example, there are significant differences between the positive and the negative half of the voltage cycle. It is, therefore, logical to record separate distributions of the negative and the positive half cycle so that four different distributions appear: for the positive half of the voltage cycle $H_{q n}^{+}(\varphi)$ and $H_{n}^{+}(\varphi)$ and for the negative half of the voltage cycle $H_{q n}^{-}(\varphi)$ and $H_{n}^{-}(\varphi)$. It has been found that these four phase-position distributions are characteristic for the type of a defect [8]. For this reason each distribution is analyzed by means of statistical operators. To evaluate the shape of $H_{q n}(\varphi)$ and $H_{n}(\varphi)$ the following operators are used.

1. Skewness $S k$, which describes the asymmetry of the $H_{q n}^{+}(\varphi), H_{q n}^{-}(\varphi), H_{n}^{+}(\varphi)$ and $H_{n}^{-}(\varphi)$ distributions with respect to a normal distribution $(S k=0$ means symmetric, $S k>0$ means asymmetric with the left side larger and $S k<0$ means asymmetric with larger right side. 

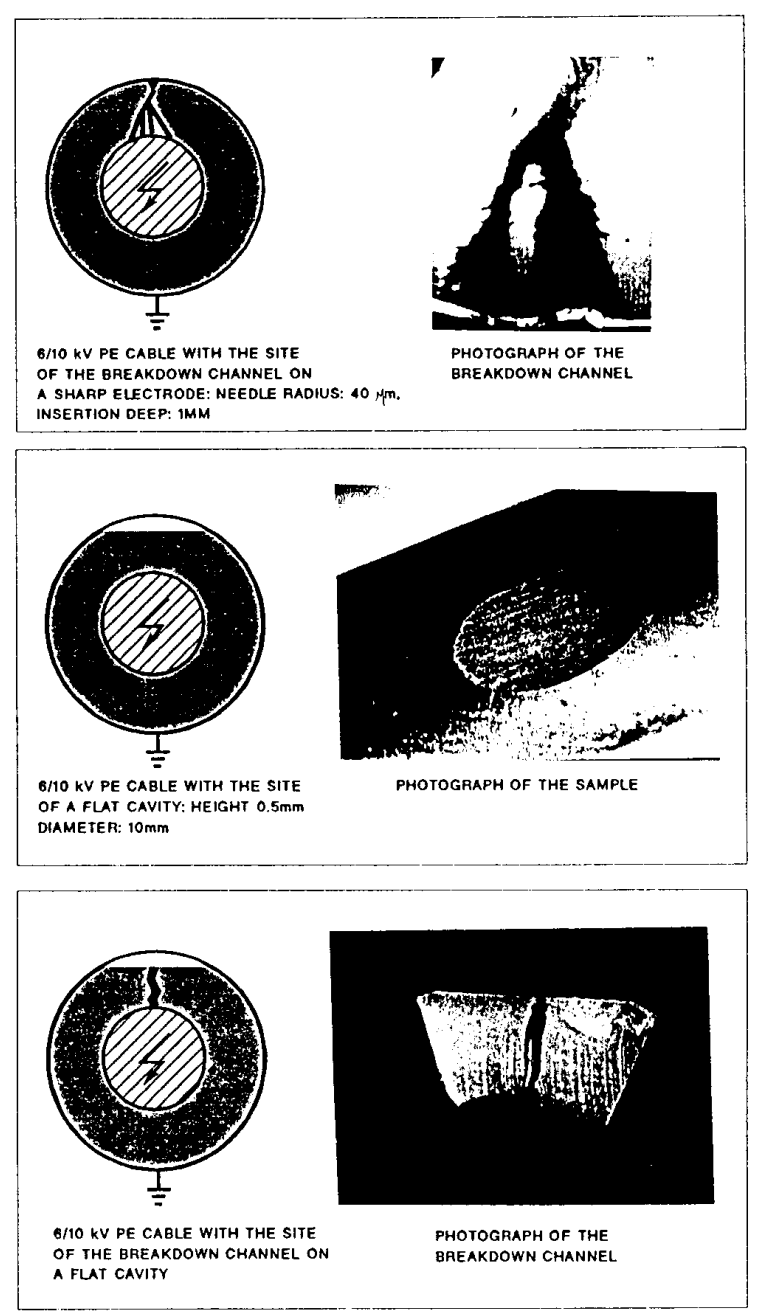

Figure 7.

Photographs of the samples and the breakdown channels in $6 / 10 \mathrm{kV} \mathrm{PE}$ cable.

2. kurtosis $K u$, which describes sharpness of the $H_{q^{n}}^{+}(\varphi)$, $H_{q n}^{-}(\varphi), H_{n}^{+}(\varphi)$ and $H_{n}^{-}(\varphi)$ distributions with respect to the normal distribution $\mathrm{Ku}=0$ means a normal distribution, $K u>0$ means a sharp distribution and $K u<0$ means a flat distribution.

3. The number of peaks $P e$ in order to distinguish between $H_{q n}^{+}(\varphi), H_{q n}^{-}(\varphi), H_{n}^{+}(\varphi)$ and $H_{n}^{-}(\varphi)$ distributions with single top and distributions with several tops (mixed distribution).

4. The cross-correlation factor $c c$, which describes the difference in shape between $H_{q n}^{+}(\varphi)$ and $H_{q n}^{-}(\varphi)$ distributions of the positive and negative half cycle. $c c=1$ means $100 \%$ shape symmetry, $c c=0$ means total asymmetry.

5. The discharge factor $Q$, which describes the difference in the mean discharge level in the $H_{g n}^{+}(\varphi)$ and $H_{q n}^{-}(\varphi)$ distributions $Q=1$ means equal discharge levels, $Q=0$ means a difference.

6. Modified cross-correlation factor mcc which is equal to product of $Q$ and $c c$.

The discharge pattern of one particular defect can now be described by the values of the 15 mentioned statistical operators above. In Figure 3(b) an example involving of these operators is shown. In previous work it has been found that each artificial defect is characterized by typical values of these operators [8]. At first, these tools appear to be purely descriptive and according to [4] they are not predictable in terms of the basic physical processes. Nevertheless, the typical phase-dependent behavior of different discharges may be studied in this way, so that these tools may be used to recognize discharges under ac conditions. In this paper the following artificial defects, as obtained in earlier work $[6,8]$ are used: cavities (different shapes and locations in the insulation); surface discharges (in air, $\mathrm{SF}_{6}$ or in oil); , corona (in air or in oil), and treeing initiated from a sharp electrode.

\subsection{STATISTICAL RECOGNITION}

Using the above described statistical tools, the characteristics of different $P D$ patterns can be quantified as shown in [6]. This makes it worth while to use such data for $\mathrm{PD}$ recognition. Generally, two questions are important here: "Can these statistical operators be used to discriminate between different defects?" and "How can the discriminating data be employed to identify discharges from unknown sources?". Nowadays different techniques are available and can be used to solve the problem of discrimination and classification: for instance, neural network techniques. Without doubt these techniques are very powerful, but is it necessary to use these techniques to analyze PD processes? The neural network techniques basically belong to nonparametric methods, so that it is usually not necessary to make any assumption about the data structure. On the other hand, it is known that the characteristics of PD may be very complex due to their dependence on a wide range of conditions at the discharge site and a strong dependence on the test time, the voltage level and even the manner of measurement $[4,9,10]$. Therefore, it is important first to use a recognition method that is related to the stochastic behavior of $\mathrm{PD}$. If positive results can be obtained, using a simple statistical method then these data may provide the basis for a more advanced method of recognition: for instance, use of classical, mathematical pattern recognition methods. 


\section{Treeing on a sharp electrode}

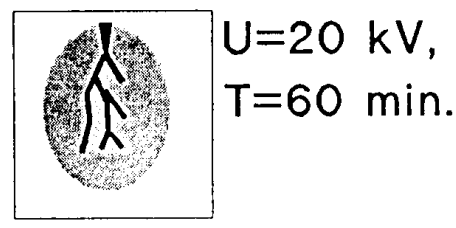

\section{Flat cavity}

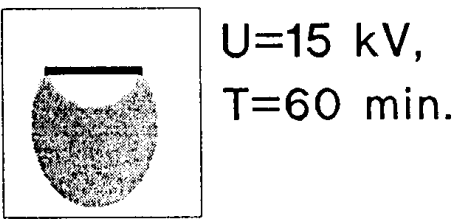

\section{Treeing on a cavity}

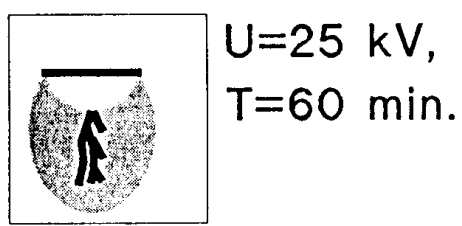

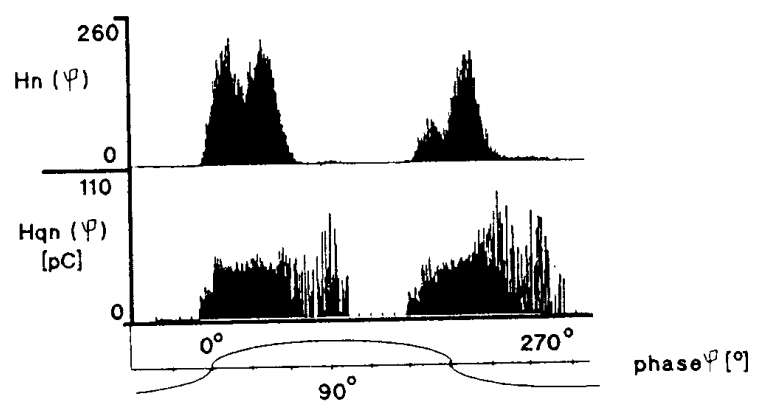
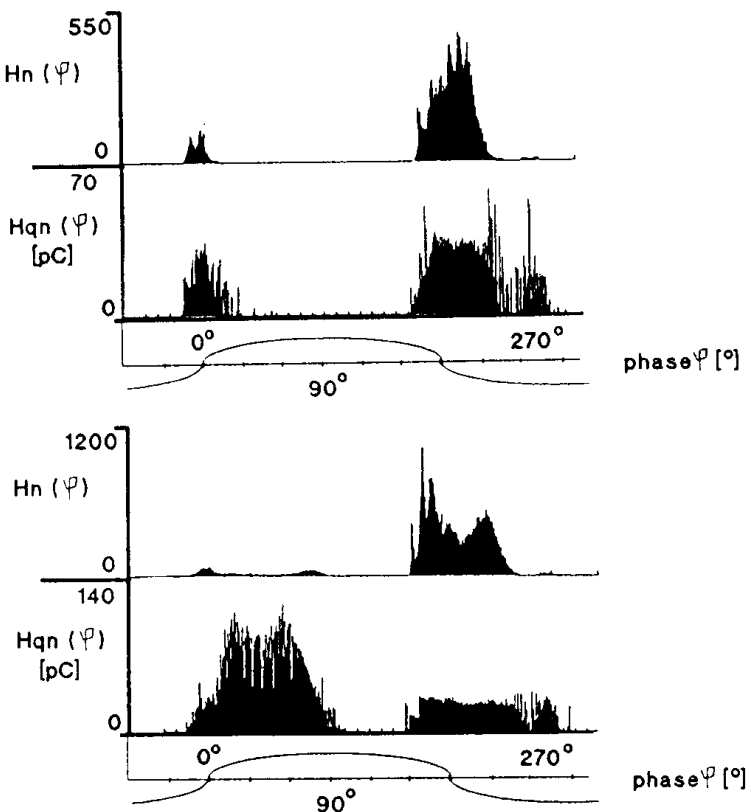

Figure 8.

Typical statistical distributions $H_{q n}(\varphi)$ and $H_{n}(\varphi)$ as observed for PD defects studied in $P E$ cable.

Therefore the main goal here was to define first a set of parameters representative for a defect i.e. the 'finger print' of a defect. For this purpose, a series of 8 to 23 observations with the same discharge source were made in order to estimate the true population of each statistical operator. It is common practice to discard a sample as belonging to the same population when the sample is found to be outside the 90 or $95 \%$ confidence interval of the true population. However, due to the fact that the estimation of these true populations are based on a relatively small number of samples the scatter, and thus the width of the distributions, will be rather large and result in a large overlap of different populations. In this situation it can be difficult to decide between two (or more) populations. The problem can be solved by using the confidence interval covering the mean of the true population. The distribution of the means of a number of observations is narrower. For each of the statistical operators, obtained with one type of defect, the mean value $M_{\text {so }}$ and the $C_{2.5 \%}$ and $C_{97.5 \%}$ quartiles for the mean value $M_{s o}$ were calculated. In this way, the $95 \%$ confidence interval covering the mean values of a statistical operators was chosen as representative and used as the finger print of the defect, see Figure 4. Examples of finger prints in the case of HV cable are shown in Figure 9. If a defect is analyzed, the analysis takes in general $20 \mathrm{~min}$, whereas 1 to $2 \mathrm{~min}$ would be sufficient to determine the statistical operators. Consequently, the mean value obtained in 20 min can be regarded as the mean of 10 to 20 values and can be compared directly with the above stated interval.

For an arbitrary discharge measurement characterized by its statistical operators $X_{o p}$, the notion recognition rate was introduced. The recognition rate represents the 


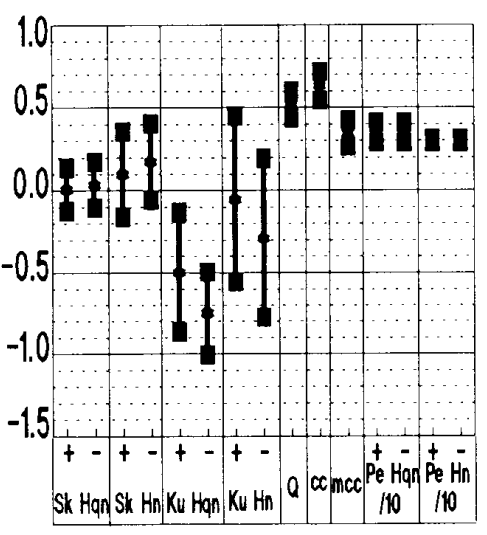

(a)

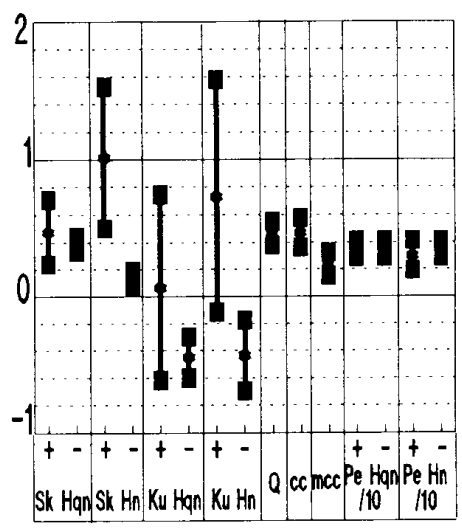

(b) $255 \quad$ mean $97.5 \%$

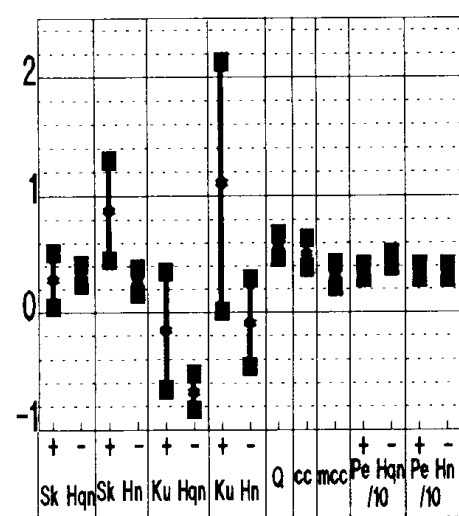

(c)

Figure 9.

The mean value and the scatters (expressed as $95 \%$ confidence intervals) of fifteen statistical operators characterizing the discharges in a particular defect. These results are obtained for tests in $6 / 10 \mathrm{kV}$ PE cable: (a) 41 tests of treeing at needle, (b) 24 tests of a flat cavity, (c) 28 tests of treeing on a cavity.

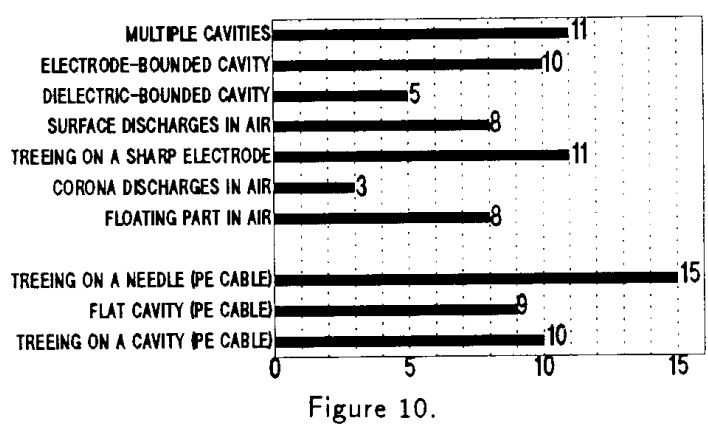

Recognition rate obtained for the test on the 6/10 kV PE cable with treeing discharges at an electrode. The defect in question represents the mean value of the statistical operators of 41 tests.

number of statistical operators that fit in the limits given by the finger print of a known discharge source. Using a matrix, the statistical operators $X_{o p}$ of an unknown defect are compared to the finger prints of the known discharge source, see Figure 5(a). The number of statistical operators (minimum 0 , maximum 15) fitting in the interval of the finger print of a defect is determined; the highest recognition rate indicates the type of defect. In this particular case, the highest recognition rate indicates that there are surface discharges in air, see Figure 5(b). Based on the highest recognition rate and the difference with other defects, the comparison between finger prints of different discharges can be made and also the indication of a possible type of defect can be discussed.

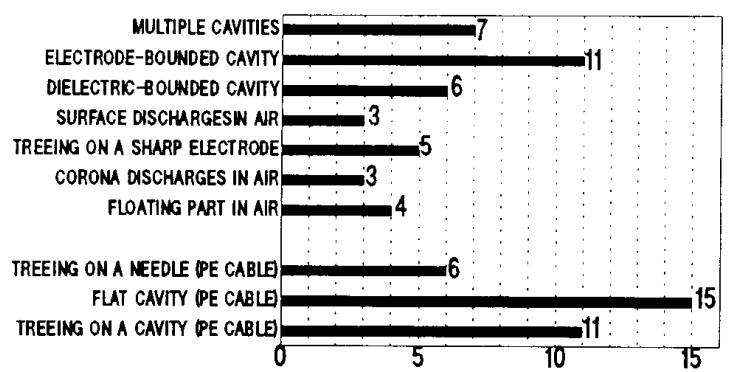

Figure 11.

Recognition rate obtained for the test on the $6 / 10$ kV PE cable with cavity discharges. The defect in question represents the mean value of the statistical operators of 24 tests.

\section{DIAGNOSIS OF HV EQUIPMENT}

The procedure described above has yielded satisfactory results in the past when describing discharges in artificial models [6]. The aim was to make statements on the type of discharges and the medium where these discharges occur in actual HV apparatus. In order to investigate typical defects following two HV devices were studied: $6 / 10 \mathrm{kV} \mathrm{PE}$ cable involving treeing propagation from a sharp electrode, flat cavity, treeing at a cavity and also in $400 \mathrm{kV} \mathrm{SF}_{6}$ insulation, involving spacer discharges, a floating part and a fixed conducting particle on a spacer.

After the tests on the above mentioned defects were 


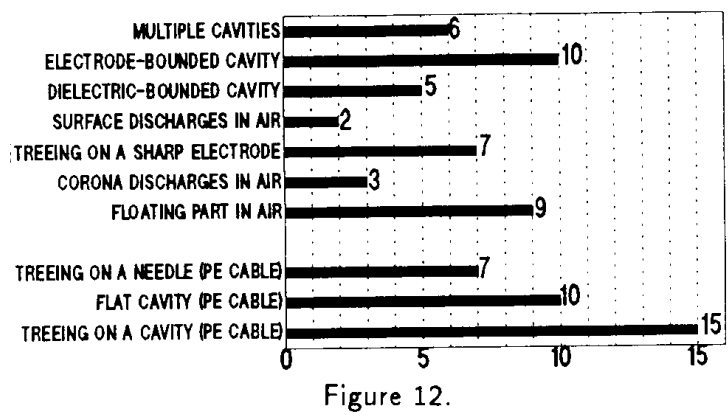

Recognition rate obtained for the test on the $6 / 10$ $\mathrm{kV}$ PE cable with treeing discharges at a cavity. The defect in question represents the mean value of the statistical operators of 28 tests.

completed, the $H_{q n}(\varphi)$ and $H_{n}(\varphi)$ distributions were analyzed by means of the 15 statistical operators. To compare the series of tests for different defects, the finger prints were obtained, i.e. the mean value of different statistical operators and the $95 \%$ confidence intervals were calculated to represent the scatter in results. An interesting question arose: are the differences between the finger prints significant? To resolve this question, the above described recognition rate algorithm was used. Each type of defect represented by the 15 mean values of statistical operators was compared to the $95 \%$ confidence intervals of model defects as shown in Figure 4 and to the practical models consisting of the $\mathrm{HV}$ cable and GIS insulation. It is evident that for the defect itself all 15 operators match the confidence intervals, resulting in total recognition. A comparison with other defects indicates how many statistical operators out of 15 of a particular defect coincide with those of another defect. For instance, if 7 out of 15 operators of an unknown defect coincide with those of multiple cavities the recognition rate against 'multiple cavities' is 7 . The highest recognition rate of 15 means no differences between both defects and the lowest recognition of 0 means the resemblance to the other defect is nil.

\subsection{ANALYSIS OF PD IN A HV CABLE}

To analyze the discharge behavior in a $\mathrm{HV}$ cable, three types of defects were studied: treeing at a sharp electrode, a flat cavity and treeing at a cavity [11]. These defects were created artificially and were placed in the middle of a $1 \mathrm{~m}$ long $6 / 10 \mathrm{kV}$ PE cable, see Figure 6 . To obtain a good screening of the measuring electrode, the electrode was covered with insulating paper and an grounded copper screen.

\subsubsection{TREEING AT A SHARP ELECTRODE}

To obtain treeing at a sharp electrode, a stainless needle with a radius of $40 \mu \mathrm{m}$ was inserted $1 \mathrm{~mm}$ deep into the insulation of the cable, a total of 10 cable specimens were employed. Starting at $15 \mathrm{kV}$, the specimens were subjected to a $5 \mathrm{kV} / \mathrm{h}$ ramp voltage until discharges $(>1 \mathrm{pC})$ were registered. After treeing was initiated the samples were subjected several times to a $60 \mathrm{~min}$. test of constant ac voltage. In total, 41 tests were carried out on 10 samples. In 7 out of 10 cables containing the sharp electrode, breakdown took place after 7 to $10 \mathrm{~h}$ aging at either 40 or $45 \mathrm{kV}$. During the time before breakdown, discharges ranging in amplitude to $50 \mathrm{pC}$ were recorded and processed as $H_{q n}(\varphi)$ and $H_{n}(\varphi)$ distributions.

\subsubsection{CAVITY DISCHARGES}

To obtain cavity discharges, an oval cavity with a $10 \mathrm{~mm}$ diameter and $0.5 \mathrm{~mm}$ depth was formed at the surface between the dielectric and semiconducting layer (see Figure 6). Ten specimens were subjected several times to a 60 min test of constant ac voltage. In total, 24 tests were carried out at a voltage level of $15 \mathrm{kV}$. During the tests no breakdowns were observed. Discharges ranging to $10 \mathrm{pC}$ were recorded and processed as $H_{q n}(\varphi)$ and $H_{n}(\varphi)$ distributions.

\subsubsection{TREEING AT A CAVITY}

To obtain treeing at a cavity, the above described 5 specimens with a flat cavity were prepared under a microscope. To initiate the treeing a needle tip of $100 \mu \mathrm{m}$ was made in the middle of the cavity. Starting at $20 \mathrm{kV}$, these specimens were subjected several times to a $60 \mathrm{~min}$ test of constant ac voltage. In total, 28 tests were carried out; in 4 out of 5 cables the breakdown took place after 5 to $7 \mathrm{~h}$ aging at either 20 or $25 \mathrm{kV}$. During the time before breakdown, discharges in range from 10 to $15 \mathrm{pC}$ were recorded and processed as $H_{q n}(\varphi)$ and $H_{n}(\varphi)$ distributions.

\subsubsection{RECOGNITION OF PD IN A HV CABLE}

Figure 7 depicts the breakdown channels as observed for PD defects in PE cables. 


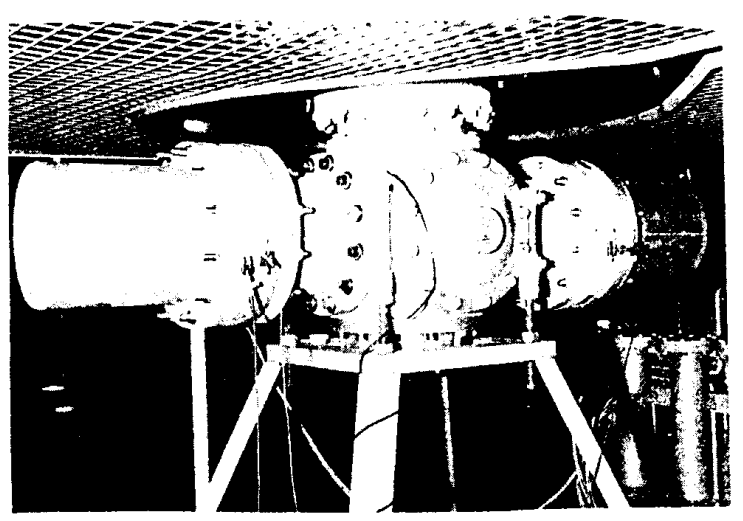

(a)

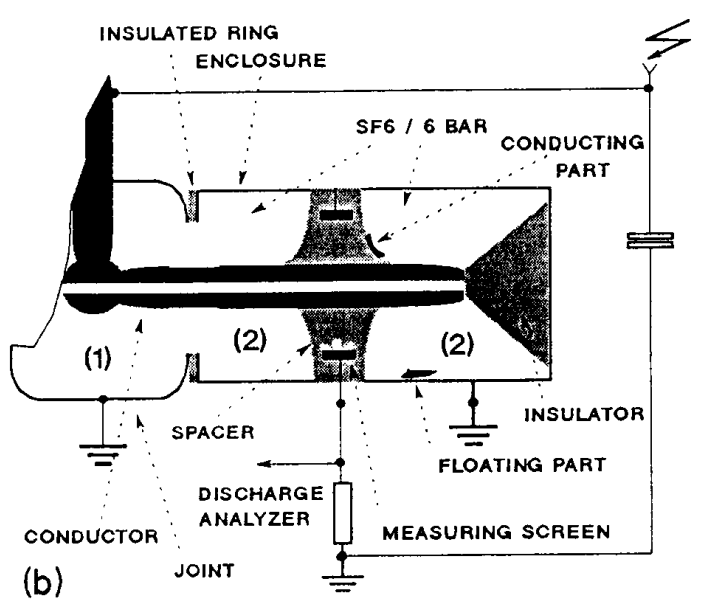

(b)

Figure 13.

Test setup for $400 \mathrm{kV} \mathrm{SF} 6$ insulation: (a) photograph of the $\mathrm{SF}_{6}$ compartments, (b) cross section of the right-hand side showing the artificial defects.

In Figure 8 typical statistical distributions obtained with the PD defects in PE cables are shown. $H_{n}(\varphi)$ gives the pulse count as a function of the phase angle $\varphi_{i}$, and $H_{q n}(\varphi)$ shows the average discharge magnitude as a function of the phase angle $\varphi_{i}$. In Figure 9 the finger prints obtained for the three defects are shown. It follows from this Figure that each defect may be characterized by a characteristic combination of 15 statistical operators.

In Figure 10 the recognition rate in the case of treeing at a sharp electrode is shown. It follows from this Figure that treeing at a needle in a PE cable resembles most closely the model defects: treeing at a sharp electrode and an electrode-bounded cavity, whereas the practical defect treeing at a cavity (PE cable) shows the highest resemblance. The small difference between the model defect, electrode-bounded cavity and treeing at a needle (PE cable) may be explained by the similarities of the discharge processes (in both cases the discharge site was adjacent to a conductor). A remarkable result is observed in the difference with defect 'treeing at a sharp electrode'. Apparently treeing discharges in a point plane configuration in Perspex ${ }^{\mathrm{TM}}$ used for the model defect of treeing are not identical with those in a PE cable, which in fact confirms the stochastic behavior of PD processes even within a series of one type of defect.

In Figure 11 the recognition rate in the case of cavity discharges is shown. It follows from this Figure that the flat cavity ( $\mathrm{PE}$ cable) differs significantly from both other practical defects and resembles best the model defect electrode-bounded cavity.
Figure 12 depicts the recognition rate of treeing at a cavity. Here too, the resemblance to other defects is limited. The defect 'electrode-bounded cavity' and the practical defect treeing at a needle exhibit a higher recognition rate as compared to other defects. These relationships may be explained by the similarities in the discharge processes of these defects.

\subsection{ANALYSIS OF PD IN GIS}

To analyze the discharges GIS installation a compartment containing joint, spacer, casing, terminal insulator and HV conductor was used, (see Figure 13) [12]. The investigations were carried out at a $\mathrm{SF}_{6}$ pressure of 0.6 $\mathrm{MPa}$. The photograph shows a wire mesh over the $\mathrm{SF}_{6}$ compartments, which screens the setup from interference at the HV side. The cross section showing the compartments is separated by insulated rings, so that the test electrodes (1) and (2) can be measured separately. Also by using the separation rings, the discharge freedom of the joint was controlled. Four types of defects have been studied.

\subsubsection{CAVITY DISCHARGES IN THE SPACER}

This defect consists of a measuring screen in a spacer where air pockets remained after casting. To measure these discharges, the joint and the casing were grounded and the measuring screen of the spacer was connected to the discharge detector. The discharges inception voltage 
Internal discharges in spacer (air pockets)

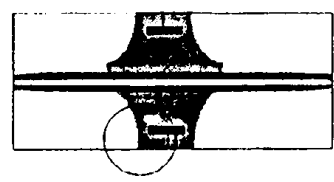

Floating part (contact spring)

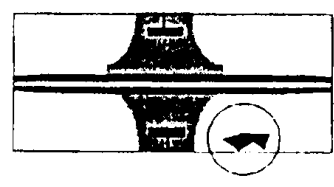

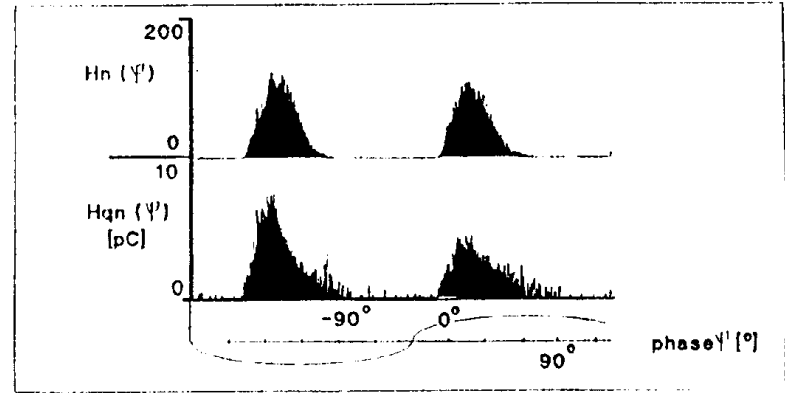
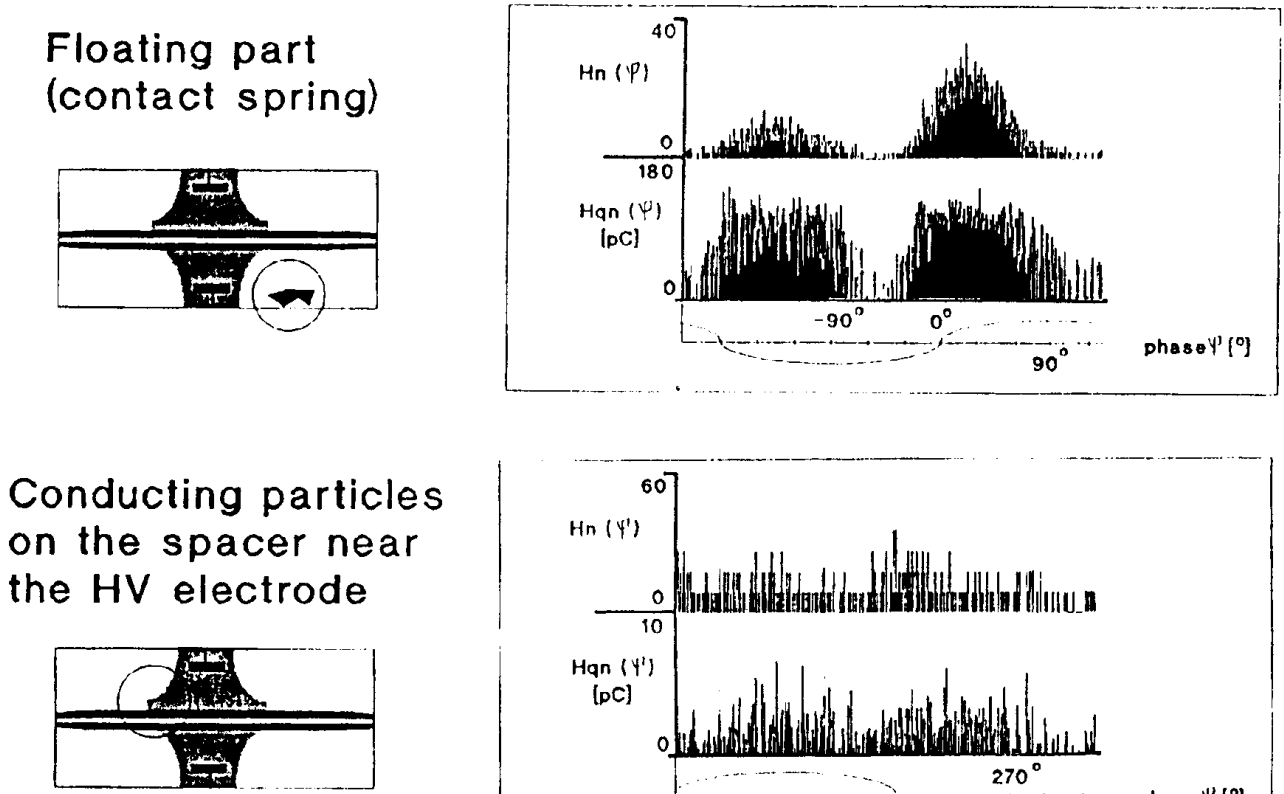

Corona discharges (Al- splinter)

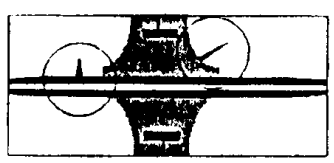

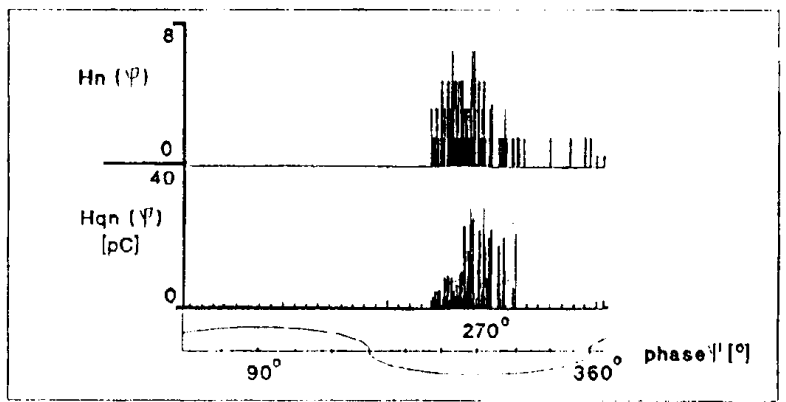

Figure 14.

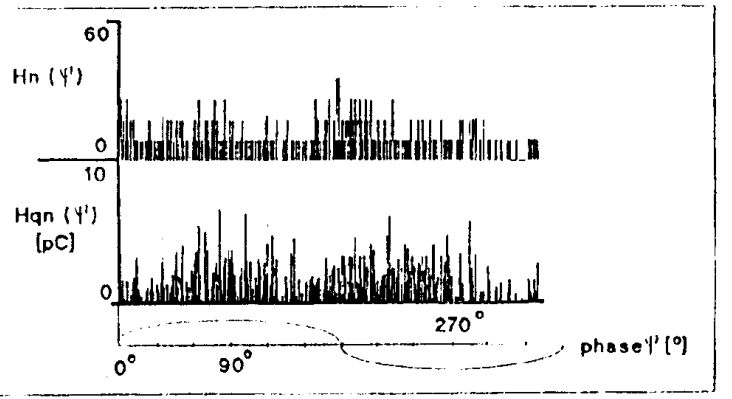

Typical statistical distributions $H_{q n}(\varphi)$ and $H_{n}(\varphi)$ as observed for PD defects studied in $400 \mathrm{kV}$ GIS compartment

was $240 \mathrm{kV}$ and the complete object was subjected to a $250 \mathrm{kV}$ test voltage for $10 \mathrm{~min}$. Discharges to $10 \mathrm{pC}$ were recorded and processed as $H_{q n}(\varphi)$ and $H_{n}(\varphi)$ distributions.

\subsubsection{FLOATING PART DISCHARGES}

This defect arises when a fallen contact spring of the circuit breaker lies at the bottom of the GIS. To measure these discharges, the joint and the measuring screen were 
Internal discharges

in spacer

(air pockets)

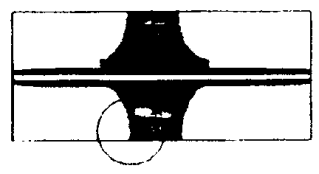

(a)

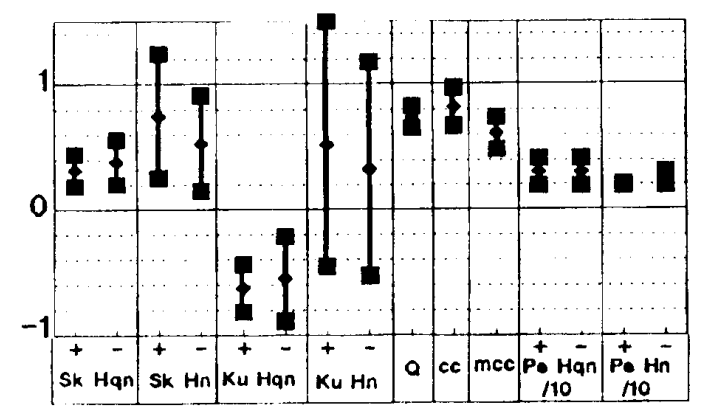

Floating part (contact spring)

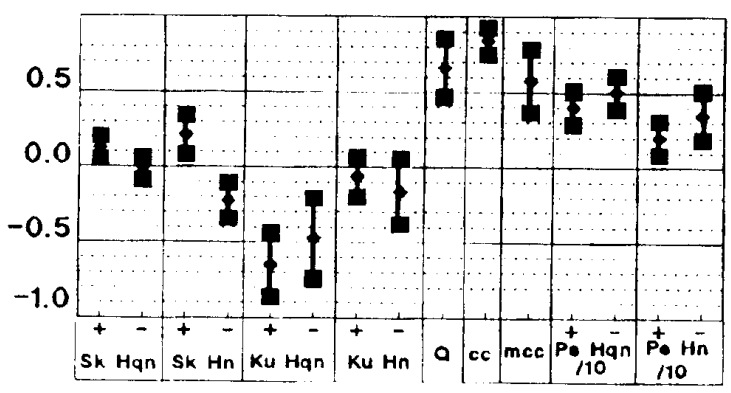

Conducting particles on the spacer near the HV electrode

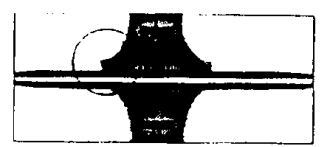

(c)

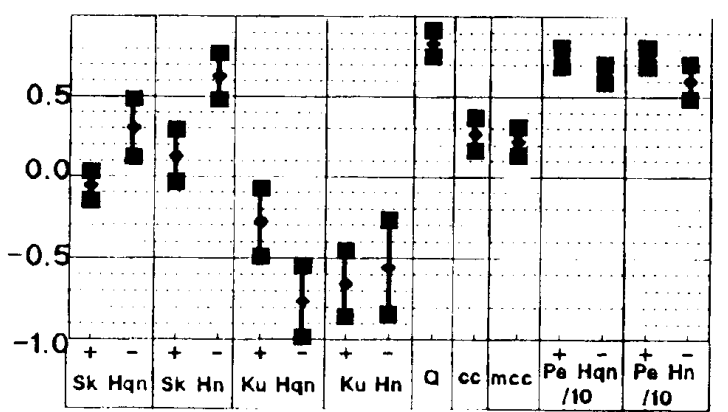

\section{Corona discharges (Al-splinter)}
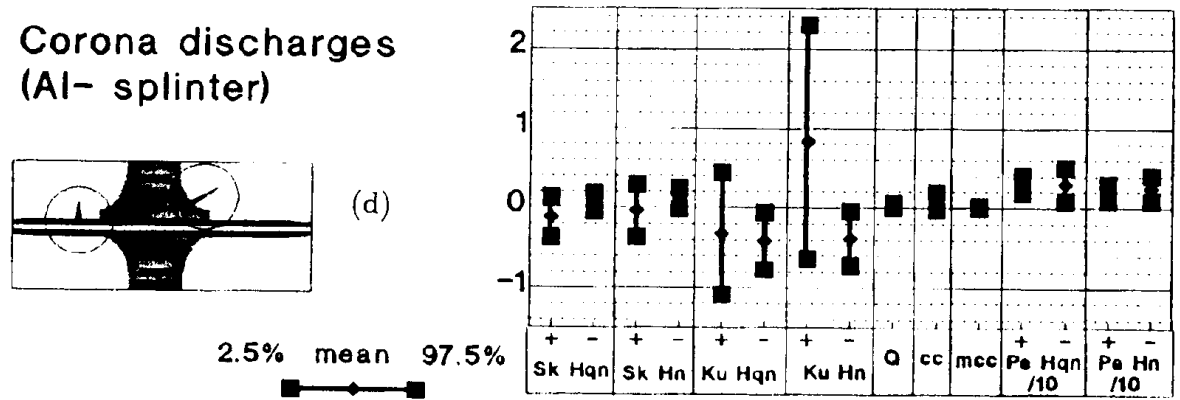

Figure 15.

The mean value and scatters (expressed as $95 \%$ confidence intervals) of fifteen statistical operators characterizing the discharges in a particular defect. These results are obtained for tests in $400 \mathrm{kV} \mathrm{SF}$ insulation: (a) 13 tests of cavity discharges in spacer, (b) 10 tests caused by a floating contact spring inside the compartment, (c) 10 tests caused by conducting particle the spacer near the HV conductor, (d) 11 tests of corona discharges inside the compartment.

grounded and the casing was connected to the discharge detector. Into the compartment under the HV conductor a GIS contact spring $\left(20 \times 5 \times 10 \mathrm{~mm}^{3}\right)$ was placed. The discharges inception voltage was $100 \mathrm{kV}$ and the com- 
plete object was subjected to a $110 \mathrm{kV}$ test voltage for 5 min. Discharges to $200 \mathrm{pC}$ were recorded and processed as $H_{q n}(\varphi)$ and $H_{n}(\varphi)$ distributions.

\subsubsection{FIXED CONDUCTING PARTICLE ON THE SPACER}

This defect consists of a conducting particle fixed on the insulating surface. To measure these discharges the joint and the measuring screen were grounded and the casing was connected to the discharge detector. On the surface of the spacer $10 \mathrm{~mm}$ from the $\mathrm{HV}$ conductor, a conducting particle $\left(3 \times 3 \times 0.1 \mathrm{~mm}^{3}\right)$ was fixed. The inception voltage of discharges was $400 \mathrm{kV}$ and the complete object was subjected to a $450 \mathrm{kV}$ test voltage for 5 min. Discharges to $8 \mathrm{pC}$ were recorded and processed as $H_{q n}(\varphi)$ and $H_{n}(\varphi)$ distributions.

\subsubsection{CORONA CAUSED BY SHARP ALUMINUM SPLINTERS}

This occur either at the conductor or at the surface of the spacer: the two varieties yielded the same recognition pattern so that they can be classified as one defect. To measure these discharges, the joint and the measuring screen were grounded and the casing was connected to the discharge detector. At the conductor or on the surface of the spacer $10 \mathrm{~mm}$ from the $\mathrm{HV}$ conductor an aluminum splinter (height $5 \mathrm{~mm}$, point radius of $100 \mu \mathrm{m}$ ) was fixed. The discharges inception voltage was $150 \mathrm{kV}$ and the complete object was subjected to a $160 \mathrm{kV}$ test voltage for $5 \mathrm{~min}$. Discharges up to $15 \mathrm{pC}$ were recorded and processed as $H_{q n}(\varphi)$ and $H_{n}(\varphi)$ distributions.

\subsubsection{RECOGNITION OF PD IN GIS}

In Figure 14 typical statistical distributions as observed for PD defects in GIS are shown: $H_{n}(\varphi)$ shows the pulse count as a function of the phase angle $\varphi_{i}$, and $H_{q n}(\varphi)$ shows the average discharge magnitude as a function of the phase angle $\varphi_{i}$.

A series of 10 to 13 tests for one type of defect were carried out and the mean value of the different statistical operators was determined as shown in Figure 15; the $95 \%$ confidence intervals were calculated to represent the scatter in the results. These sets of operators may serve as a finger print of the three defects studied in GIS. As basis for comparison, similar finger prints were used for artificial defects in the models as mentioned above. In Figure 16 the recognition rate in the case of cavity discharges in the spacer is shown. It follows from this Figure that cavity discharges in the spacer are well recognizable and resemble best the model defect i.e. dielectric-bounded cavity, whereas the resemblance to practical defects in GIS is nil.

In Figure 17 the recognition rate of the floating part is recorded. It follows from this Figure that discharges caused by a floating part of this size can be clearly discriminated from other GIS defects. In contrast to the results presented in [12] (6 of 15 statistical operators coincide) the resemblance to floating part in air is evident: 10 of 15 statistical operators coincide. This confirms, as in the case of treeing discharges in $\mathrm{HV}$ cables, the complexity of PD processes even within a series of one type of defect. Thus for some defects no unambiguous finger print can be found.

In Figure 18 the recognition rate of the fixed conducting particle on the spacer is recorded. It follows from this Figure that discharges caused by this defect differ very clearly from all model defects and from all practical defects in the GIS studied. This low resemblance to other defects may be caused by the asymmetry between the $\mathrm{PD}$ behavior on the positive and the negative half cycles of the voltage which can be effectively delineated by the statistical operators used [13].

In Figure 19 the recognition rate of the corona discharges is recorded. It follows from this Figure that discharges caused by this defect differ very clearly from model defects and from practical defects in the GIS studied. Hence, the corona discharges in GIS have some likeness to the model defect corona discharges in air, as might be expected.

\section{CONCLUSIONS}

The results of this first attempt to evaluate discharges in actual $\mathrm{HV}$ devices using statistical tools are modest but encouraging. It has been shown that phase-resolved PD analysis as a complementary tool to conventional PD detection provides additional information about discharge sources. Because of the stochastic behavior of discharges in general much more works (more defects as well as extensive testing) is needed to investigate the validity of this method; the encouraging results of this study makes these efforts worth while. 


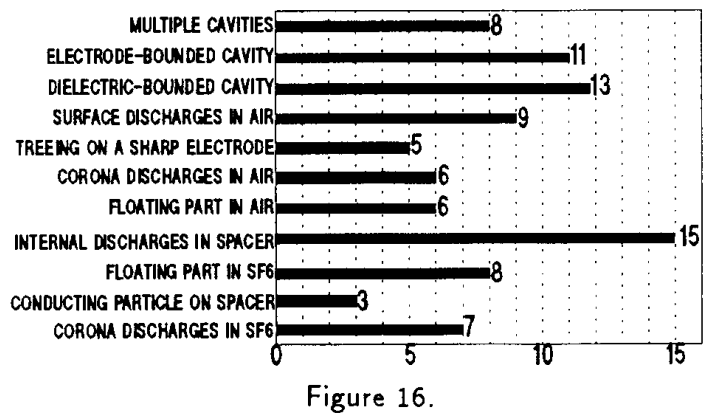

Recognition rate of the discharges in the $400 \mathrm{kV}$ spacer. The defect in question represents the mean value of the statistical operators of 13 tests.

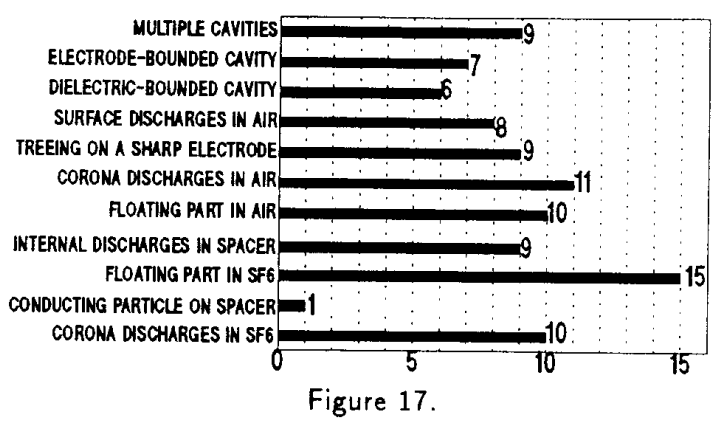

Recognition rate of floating part discharges in $400 \mathrm{kV}$ GIS. The defect in question represents the mean value of the statistical operators of 10 tests.

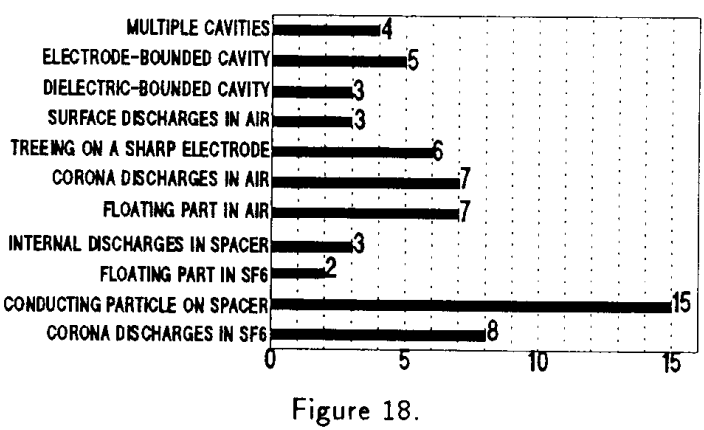

Recognition rate of fixed conducting particle on the spacer discharges in $400 \mathrm{kV}$ GIS. The defect in question represents the mean value of the statistical operators of 10 tests.

\section{REFERENCES}

[1] CIGRE Working Group 21.03 "Recognition of Discharges", Electra, Vol. 11, pp. 61-98, 1969.

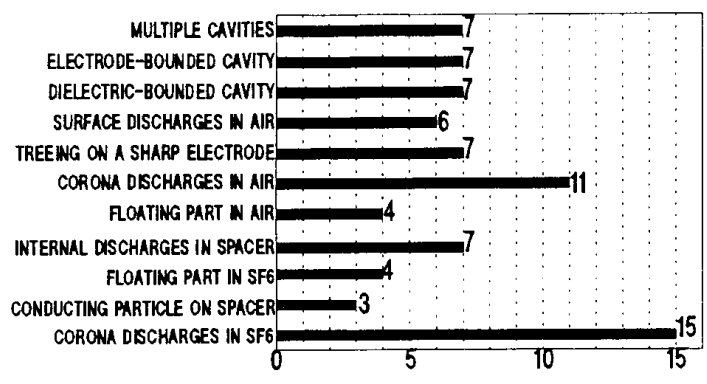

Figure 19.

Recognition rate of corona discharges in $400 \mathrm{kV}$ GIS. The defect in question represents the mean value of the statistical operators of 11 tests.

[2] B. H. Ward, "Digital Techniques for Partial Discharge Measurements", IEEE Trans. Power Delivery, Vol. 7, pp. 469-479, 1992.

[3] W. Hutter, "Partial Discharge Detection in Rotating Electrical Machines", IEEE Electrical Insulation Magazine, Vol. 8, pp. 21-32, 1992.

[4] R. J. Van Brunt, "Stochastic Properties of Partial Discharge Phenomena", IEEE Trans. Elec. Insul., Vol. 26, pp. 902-948, 1991.

[5] E. Neudert, R. Porzel, "Ein oszillografisches Verfahren zur Beurtelung von Teilentladungen", Elektrie, Vol. 9, pp. 360-362, 1969.

[6] E. Gulski, Computer-aided recognition of partial discharges using statistical tools, Delft University Press, pp. 15-61, 143-158, 1991.

[7] P. Seitz "Permanent Digital Recording of PD Impulses in an Impulse Analyzer", Proc. 5th Haefely Symp., Stuttgart, paper 4.1, 1992.

[8] E. Gulski and F. H. Kreuger, "Computer-aided Recognition of Discharge Sources", IEEE Trans. Elec. Insul., Vol. 27, pp. 82-92, 1992.

[9] P. H. F. Morshuis and F. H. Kreuger, "The Spatial Distribution and Electrical Parameters of PD in Polyethylene Insulation During Aging", Conf. Rec. 4th Int. Conf. on Conduction and Breakdown in Solid Dielectrics, IEEE Publ. 92 CH3034-6, pp. 209-214, 1992.

[10] E. Gulski and F. H. Kreuger, "Determination of Discharge Sources by Analysis of Discharge Quantities as a Function of Time", Conf. Rec. 1992 IEEE Int. Symp. on Elec. Insul., IEEE Publ. 92 CH3150-06, pp. 397-400, 1992. 
[11] E. Gulski, P. H. F. Morshuis and J. Janssen, "Recognition of Defects in H. V. Cables Using Statistical Tools", Conf. Rec. 4th Int. Conf. on Conduction and Breakdown in Solid Dielectrics, IEEE Publ. 92 CH3034-6, pp. 140-144, 1992.

[12] F. H. Kreuger, E. Gulski and W. A. Sonneveld, "Diagnosis in GIS by Statistical Analysis of Discharges", Proc. CIGRE Conf.,Paris September 1992.
[13] E. Gulski, P. H. F. Morshuis and F. H. Kreuger, "Automized Recognition of Partial Discharges in Cavities", Japanese Journal of Applied Physics, Vol. 29, pp. 1329-1335, 1990.

This paper is based on a presentation given at the 1992 Volta Colloquium on Partial Discharge Measurements, Como, Italy, 26-28 A ugust 1992.

Manuscript was received on 21 October 1992, in revised form 20 January 1993. 